\title{
Testing of diabetes-associated WFS1 polymorphisms in the Diabetes Prevention Program
}

\author{
J. C. Florez • K. A. Jablonski • J. McAteer • \\ M. S. Sandhu • N. J. Wareham • I. Barroso • \\ P. W. Franks • D. Altshuler - W. C. Knowler • \\ for the Diabetes Prevention Program Research Group
}

Received: 20 August 2007 / Accepted: 30 October 2007 / Published online: 4 December 2007

(C) Springer-Verlag 2007

\begin{abstract}
Aims/hypothesis Wolfram syndrome (diabetes insipidus, diabetes mellitus, optic atrophy and deafness) is caused by mutations in the WFS1 gene. Recently, single nucleotide polymorphisms (SNPs) in WFS1 have been reproducibly associated with type 2 diabetes. We therefore examined the effects of these variants on diabetes incidence and response
\end{abstract}

Electronic supplementary material The online version of this article (doi:10.1007/s00125-007-0891-x) contains supplementary material, which is available to authorised users.

J. C. Florez $\cdot$ J. McAteer $\cdot$ D. Altshuler

Center for Human Genetic Research,

Massachusetts General Hospital,

Boston, MA, USA

J. C. Florez $\cdot$ D. Altshuler

Department of Medicine,

Massachusetts General Hospital,

Boston, MA, USA

J. C. Florez $\cdot$ D. Altshuler

Department of Medicine, Harvard Medical School,

Boston, MA, USA

J. C. Florez $\cdot$ J. McAteer $\cdot$ D. Altshuler

Program in Medical and Population Genetics,

Broad Institute of Harvard and MIT,

Cambridge, MA, USA

K. A. Jablonski

The Biostatistics Center, George Washington University, Rockville, MD, USA

M. S. Sandhu $\cdot$ N. J. Wareham

MRC Epidemiology Unit, Strangeways Research Laboratory,

Cambridge, UK

M. S. Sandhu

Department of Public Health and Primary Care, Institute of Public Health, University of Cambridge,

Cambridge, UK to interventions in the Diabetes Prevention Program (DPP), in which a lifestyle intervention or metformin treatment was compared with placebo.

Methods We genotyped the WFS1 SNPs rs10010131, rs752 854 and rs734312 (H611R) in 3,548 DPP participants and performed Cox regression analysis using genotype, intervention and their interactions as predictors of diabetes

I. Barroso

Metabolic Disease Group, Wellcome Trust Sanger Institute,

Hinxton, UK

P. W. Franks

Genetic Epidemiology and Clinical Research Group, Institute of Public Health and Clinical Medicine,

Umeå University Hospital,

Umeå, Sweden

D. Altshuler

Department of Genetics, Harvard Medical School,

Boston, MA, USA

W. C. Knowler

Diabetes Epidemiology and Clinical Research Section,

National Institute of Diabetes and Digestive and Kidney Diseases,

Phoenix, AZ, USA

J. C. Florez $(\square)$

c/o Diabetes Prevention Program Coordinating Center,

The Biostatistics Center, George Washington University,

6110 Executive Blvd., Suite 750,

Rockville, MD 20852, USA

e-mail: jcflorez@partners.org 
incidence. We also evaluated the effect of these SNPs on insulin resistance and beta cell function at 1 year.

Results Although none of the three SNPs was associated with diabetes incidence in the overall cohort, white homozygotes for the previously reported protective alleles appeared less likely to develop diabetes in the lifestyle arm. Examination of the publicly available Diabetes Genetics Initiative genome-wide association dataset revealed that rs 10012946 , which is in strong linkage disequilibrium with the three WFS1 SNPs $\left(r^{2}=0.88-1.0\right)$, was associated with type 2 diabetes (allelic odds ratio $0.85,95 \%$ CI $0.75-0.97$, $p=0.026$ ). In the DPP, we noted a trend towards increased insulin secretion in carriers of the protective variants, although for most SNPs this was seen as compensatory for the diminished insulin sensitivity.

Conclusions/interpretation The previously reported protective effect of select WFS1 alleles may be magnified by a lifestyle intervention. These variants appear to confer an improvement in beta cell function.

Keywords Beta cell function - Diabetes prevention - Genetic association study . Single nucleotide polymorphism .

Type 2 diabetes · Wolfram syndrome

$\begin{array}{ll}\text { Abbreviations } \\ \text { DPP } & \begin{array}{l}\text { Diabetes Prevention Program } \\ \text { homeostasis model assessment of insulin } \\ \text { HOMA-IR }\end{array} \\ \text { HR } & \begin{array}{l}\text { hazard ratio } \\ \text { HWE }\end{array} \\ \text { Hardy-Weinberg equilibrium } \\ \text { LD } & \text { linkage disequilibrium } \\ \text { OR } & \begin{array}{l}\text { odds ratio } \\ \text { single nucleotide polymorphism }\end{array}\end{array}$

\section{Introduction}

The search for common type 2 diabetes genes has followed one of two general strategies: a comprehensive scan of the entire genome, which is indifferent to biological function, or a specific test of association for selected candidate genes. The former, originally performed through linkage approaches, has only recently achieved the desired balance in polymorphism density, statistical power and affordable cost to be practicable via tests of association. Thus, investigators have traditionally compiled lists of candidate genes from various lines of available evidence. In this regard, monogenic syndromes of glucose intolerance transmitted in a Mendelian fashion provide theoretically attractive candidate genes: the expectation is that polymorphisms in those genes that have a less radical effect on function than the known index mutations may cause a less dramatic form of diabetes [1].
One such entity is Wolfram syndrome (OMIM no. 222300), which gives rise to diabetes insipidus, diabetes mellitus, optic atrophy and deafness. Onset occurs at 6-8 years of age and the outcome is often fatal. The clinical manifestations result from progressive degeneration of sensory neurons and pancreatic beta cells. The culprit mutations, transmitted in an autosomal recessive fashion, have been localised to the WFS1 gene by positional cloning. WFS1, located on chromosome 4p16, encodes wolframin, a $100 \mathrm{kDa}$ transmembrane protein, which is expressed in neurons and pancreatic beta cells and regulates calcium fluxes in the endoplasmic reticulum [2].

WFS1 was included in a list of 84 candidate genes recently evaluated for association with type 2 diabetes in a set of four white case-control populations [3]. A total of 1,536 single nucleotide polymorphisms (SNPs) were genotyped in a twostage approach, with two of 18 SNPs originally associated with type 2 diabetes achieving replication in the second stage. The two SNPs, rs 10010131 and rs6446482, were in strong linkage disequilibrium (LD) with each other $\left(r^{2}=0.98\right)$ and both were located in WFS1. Fine-mapping of the region identified a correlated third intronic SNP (rs752854) as well as a missense SNP (rs734312), which codes for an $\mathrm{R} \rightarrow \mathrm{H}$ change at position 611 of wolframin (different from previously described Wolfram syndrome mutations). All four SNPs were strongly associated with type 2 diabetes in an expanded set of seven populations, comprising 9,533 patients and 11,389 control persons. The association was statistically robust ( $p=1.4 \times 10^{-7}$ for the best SNP, rs10010131) but modest (allelic odds ratio [OR] 0.90, 95\% CI 0.86-0.93), with the minor allele conferring protection against type 2 diabetes.

In order to better characterise the phenotypic effects of these variants, assess their impact on diabetes incidence, extend these observations to other populations and assess whether genotype at this locus impacts on the effectiveness of diabetes preventive interventions, we genotyped three of the WFS1 SNPs in the Diabetes Prevention Program (DPP) [4].

\section{Methods}

The DPP The DPP enrolled 3,234 US participants at high risk of developing diabetes (on the basis of overweight, increased fasting glucose and impaired glucose tolerance) and randomised them to placebo, metformin $850 \mathrm{mg}$ twice daily or a lifestyle intervention aimed at $\geq 7 \%$ weight loss and $\geq 150$ min of physical activity per week; a fourth arm of 585 participants initially randomised to troglitazone was terminated early because of concerns with hepatotoxicity [4]. The main endpoint was development of diabetes confirmed by OGTT. The trial was conducted at 27 centres, all of which obtained individual Institutional Review Board approval. The DPP showed that participants treated with metformin or with a lifestyle intervention were $31 \%$ or $58 \%$ 
less likely to develop diabetes after an average of 3 years of follow-up, respectively [4].

The 3,548 DPP participants presented here (2,994 who completed the trial in the placebo, metformin or lifestyle arms, plus 554 originally randomised to troglitazone) provided informed consent specific to genetic investigation. The distribution of self-reported ethnicities among participants in this genetic study was $56.4 \%$ white, $20.2 \%$ African American, 16.8\% Hispanic, 4.3\% Asian and 2.4\% American Indian. The mean age was 51 years and mean BMI was $34.0 \mathrm{~kg} / \mathrm{m}^{2}$.

Quantitative glycaemic traits The baseline and 1-year OGTTs were used to calculate measures of beta cell function and insulin sensitivity as previously described [5]. The insulinogenic index was calculated as: ([insulin at $30 \mathrm{~min}]-$ [insulin at $0 \mathrm{~min}]) /([$ glucose at $30 \mathrm{~min}]-$ [glucose at $0 \mathrm{~min}$ ]). The insulin sensitivity index (reciprocal of insulin resistance by the homeostasis model assessment of insulin resistance [HOMA-IR]) was calculated as described previously [5].

SNP selection and genotyping We attempted to genotype the three SNPs shown to have statistically robust associations in the original report (rs10010131, rs6446482 and rs752854) [3], as well as the missense SNP rs734312 (R611H). Genotyping was initially performed by allele-specific primer extension of single-plex amplified products, with detection by matrixassisted laser desorption ionisation-time of flight mass spectroscopy on a Sequenom platform (San Diego, CA, USA), as previously described [5]. After two separate genotyping attempts, rs10010131 and rs6446482 continued to fail Hardy-Weinberg equilibrium (HWE) in the white subpopulation when scored by the automatic Sequenom genotype-calling algorithm. Visual inspection of the traces revealed preferential heterozygote dropout for these two SNPs. Manual correction of genotypes achieved HWE for both SNPs, while computerised clustering did so for rs10010131 only. To confirm the genotypes assigned by the computerised clustering algorithm, we re-genotyped rs10010131 on a TaqMan platform (Applied Biosystems, Foster City, CA, USA): concordance between clustered Sequenom and TaqMan genotypes was $98.8 \%$, with genotyping success rates of $98.9 \%$ on Sequenom and $99.7 \%$ on TaqMan (when genotypes were discordant between both platforms, a null genotype was assigned to that sample). Because of lingering concerns about genotype quality for SNP rs6446482 and its very strong LD with rs10010131 $\left(r^{2}=\right.$ 0.96 and 1.0 in HapMap Europeans and Africans, respectively), this SNP was not examined further.

Statistical analysis The primary endpoint was time to onset of diabetes. We examined Cox regression models with genotype, intervention and genotype-intervention interactions as the independent variables predicting time to diabetes. We performed analyses based on three separate genotypic groups for each SNP as well as the additive genetic model. For the quantitative trait analyses, we used general linear models to compare baseline and 1 year measures in the entire cohort according to genotype at each SNP. All analyses were repeated in white participants only. Because this study represented an attempt to replicate and further characterise a previously established finding, a $p$ value of $<0.05$ was considered statistically significant.

For power calculations of diabetes incidence within each treatment arm, we assumed HWE within each ethnic group, a homogeneous genetic effect across ethnic groups and an additive genetic model; for the overall cohort, we further assumed no interaction of genotype with intervention [6]. These calculations show that the overall DPP cohort has $54 \%$ power to detect the previously reported effect size of $\sim 0.9$ for a SNP of $40 \%$ frequency; the placebo arm has only $31 \%$ power.

\section{Results}

There were no statistically significant interactions between genotype and DPP intervention for any of the three WFS1 SNPs. None showed a statistically significant effect on diabetes incidence in the full cohort, although in the lifestyle arm hazard ratios (HRs) for participants carrying two copies of the minor allele were consistent with protection from diabetes, with $95 \%$ CI overlapping the point estimates previously reported in cross-sectional casecontrol samples (Table 1). This apparent protection achieved nominal statistical significance for white minor allele homozygotes at SNP rs752854 (HR 0.30, 95\% CI $0.09-0.99, p=0.048$ ). Analyses in white participants under the additive model showed comparable HR in the lifestyle arm $(0.72-0.88)$ but did not reach nominal significance $(p=$ $0.07-0.42)$.

Several SNPs showed reciprocal effects on insulin secretion and insulin sensitivity. For example, at baseline minor allele homozygotes at SNP rs734312 had a higher insulinogenic index $(p=0.02)$, but this could be interpreted as an appropriate compensatory response to their nominally lower insulin sensitivity ( $p=0.04$; Table 2 ). After 1 year of lifestyle intervention, a similar phenomenon was noted for the same SNP in the full cohort (Table 3) and for all three SNPs in white participants only (Electronic supplementary material [ESM] Tables 1, 2).

This seemingly compensatory effect was uncoupled for SNP rs734312 in the metformin arm: minor allele homozygotes showed a higher insulinogenic index at 1 year than 


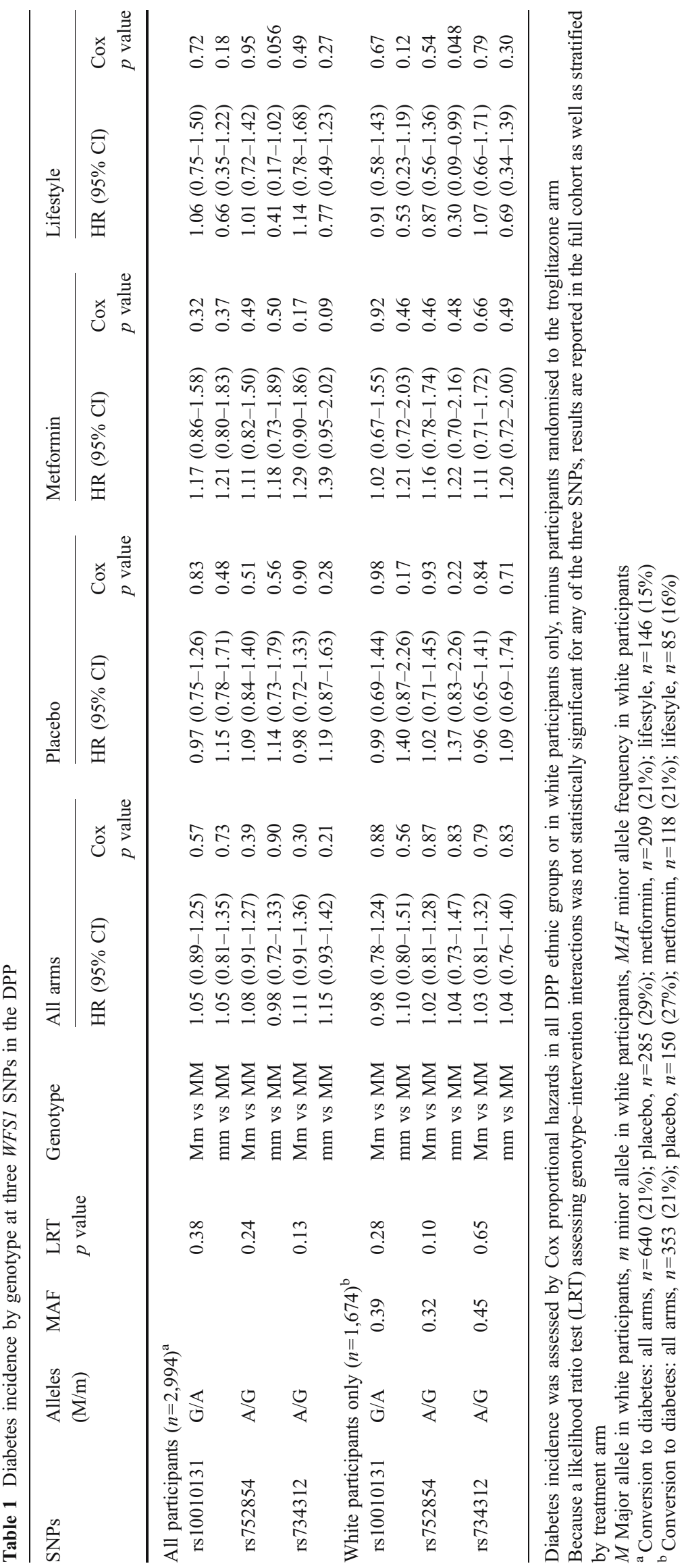


Table 2 Baseline measures of insulin sensitivity and beta cell function by WFS1 SNP in all DPP participants

\begin{tabular}{|c|c|c|c|c|c|c|c|}
\hline \multirow[t]{3}{*}{ Variable } & \multirow[t]{3}{*}{ Genotype } & \multicolumn{2}{|l|}{ rs 10010131} & \multicolumn{2}{|l|}{ rs 752854} & \multicolumn{2}{|l|}{ rs 734312} \\
\hline & & \multicolumn{2}{|l|}{$(n=3,549)$} & \multicolumn{2}{|l|}{$(n=3,535)$} & \multicolumn{2}{|l|}{$(n=3,530)$} \\
\hline & & Mean $(95 \% \mathrm{CI})$ & $p$ value & Mean $(95 \% \mathrm{CI})$ & $p$ value & Mean $(95 \% \mathrm{CI})$ & $p$ value \\
\hline \multirow[t]{3}{*}{ Insulin sensitivity index } & AA & $0.157(0.149-0.166)$ & \multirow[t]{3}{*}{0.07} & $0.166(0.162-0.171)$ & \multirow[t]{3}{*}{0.44} & $0.170(0.165-0.177)$ & \multirow[t]{3}{*}{0.04} \\
\hline & $\mathrm{AG}$ & $0.163(0.159-0.168)$ & & $0.162(0.157-0.167)$ & & $0.164(0.159-0.168)$ & \\
\hline & GG & $0.168(0.163-0.173)$ & & $0.162(0.152-0.172)$ & & $0.160(0.155-0.165)$ & \\
\hline \multirow[t]{3}{*}{ Insulinogenic index } & AA & $142.9(133.6-152.3)$ & \multirow[t]{3}{*}{0.76} & $146.4(141.8-150.9)$ & \multirow[t]{3}{*}{0.68} & $141.9(135.6-148.3)$ & \multirow[t]{3}{*}{0.02} \\
\hline & $\mathrm{AG}$ & $144.1(139.0-149.2)$ & & $143.1(137.6-148.7)$ & & $141.6(136.4-146.8)$ & \\
\hline & GG & $146.2(141.2-151.3)$ & & $144.9(133.3-156.6)$ & & $152.4(146.3-158.6)$ & \\
\hline
\end{tabular}

The insulin sensitivity index (reciprocal of insulin resistance by HOMA-IR) and the insulinogenic index ([pmol/1]/[mmol/1]) were estimated in DPP participants at baseline

Log-transformed baseline measures were compared across genotypes by ANOVA ( $F$ test); untransformed means are presented

The previously reported protective alleles are A for rs10010131 and G for both rs752854 and rs734312

their heterozygous or major allele homozygous counterparts, despite similar levels of insulin sensitivity (Table 3).

Finally, we examined publicly available genome-wide datasets for SNPs in this region. In the Diabetes Genetics Initiative [7] (http://www.broad.mit.edu/diabetes, last accessed in November 2007), SNP rs10012946, which is in strong LD with the three WFS1 SNPs $\left(r^{2}=0.88-1.0\right)$, was associated with type 2 diabetes (allelic OR $0.85,95 \%$ CI 0.75-0.97, $p=0.026$ ). The diabetic samples for the UK Wellcome Trust Case Control Consortium [8] (http://www. wtccc.org.uk, last accessed in November 2007) had already been studied in the original report that explored this gene [3]; not surprisingly, results for SNP rs10012946 were consistent with those reported for the four WFS1 SNPs analysed previously (allelic OR $0.93,95 \%$ CI $0.85-1.01$, $p=0.08$ ).

\section{Discussion}

Well-powered replication attempts and, more recently, genome-wide association scans have generated a growing list of reproducible diabetes genes (reviewed in [9]). A recent report that achieved similar levels of statistical evidence for SNPs in WFS1 [3], coupled with consistent results from independent [7] and overlapping [8] genomewide association scans, as well as the data presented here and in an accompanying report in this issue [10], confirm that WFS1 should join that expanding list as a genuinely novel type 2 diabetes gene.

The results we have obtained in the DPP, while consistent with the previous report, only achieved marginal statistical significance. This could be due, as suggested by our power calculations, to lack of power (particularly when analyses were restricted to a single ethnic group or treatment arm). Other likely factors include: (1) the ethnic heterogeneity in our cohort; (2) its starting point as a subgroup with altered glycaemic physiology at baseline; and (3) a clinical trial design in which the intervention arms were specifically intended to diminish the number of incident events. Nevertheless, consistent genetic effects were detected in the lifestyle arm.

Although the reciprocal effects of these SNPs on measures of insulin secretion and insulin sensitivity precluded us from drawing strong conclusions as to their mechanism(s) of action, the few settings in which the protective allele increased insulin secretion in the absence of decreased insulin sensitivity suggest that these variants act on the pancreatic beta cell; such a model is consistent with what is known about the pattern of expression of wolframin and the pathophysiology of Wolfram syndrome. A more detailed characterisation may require more sensitive measures of insulin secretion and sensitivity, although given the modest impact of these variants, a very large sample will be required.

Given the modest OR reported for most novel diabetesassociated variants identified in recent genome-wide association studies [9] and the number of samples required to detect true effects in case-control designs [7, 8], studies seeking to confirm or extend these observations will need to account for possible type II error. This may be even more pertinent for population-based studies (particularly if short in duration or ethnically heterogeneous), as well as for clinical trials powered to demonstrate a significant impact of an intervention, but not necessarily an interaction with a genetic variant of weak effect.

In conclusion, we present evidence that supports the role of common variants in WFS1 as modest contributors to diabetes risk and suggest that they may do so by conferring an impairment in insulin secretion. 


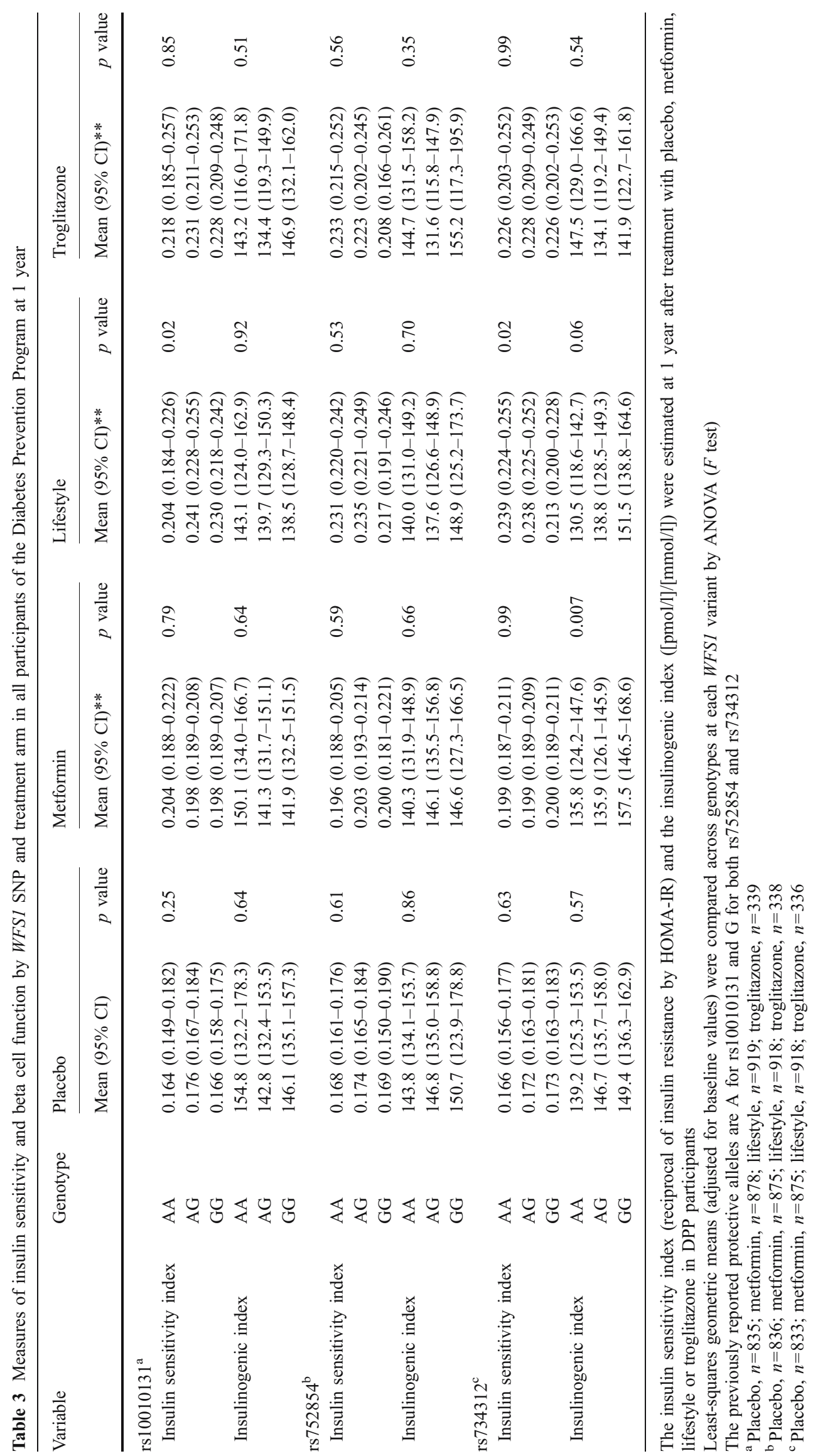


Acknowledgements The National Institute of Diabetes and Digestive and Kidney Diseases (NIDDK) of the National Institutes of Health (NIH) provided funding to the clinical centres and the Coordinating Center for the design and conduct of the study, as well as the collection, management, analysis and interpretation of the data. The Southwestern American Indian Centers were supported directly by the NIDDK and the Indian Health Service. The General Clinical Research Center Program, National Center for Research Resources supported data collection at many of the clinical centres. Funding for data collection and participant support was also provided by the Office of Research on Minority Health, the National Institute of Child Health and Human Development, the National Institute on Aging, the Centers for Disease Control and Prevention, the Office of Research on Women's Health and the American Diabetes Association. BristolMyers Squibb and Parke-Davis provided medication. This research was also supported, in part, by the intramural research program of the NIDDK. LifeScan, Health O Meter, Hoechst Marion Roussel, MerckMedco Managed Care, Merck, Nike Sports Marketing, Slim Fast Foods and Quaker Oats donated materials, equipment or medicines for concomitant conditions. McKesson BioServices, Matthews Media Group and the Henry M. Jackson Foundation provided support services under subcontract with the Coordinating Center. The opinions expressed are those of the investigators and do not necessarily reflect the views of the Indian Health Service or other funding agencies.

This work was funded by grant no. R01 DK072041-02 to D. Altshuler and J. C. Florez, who is also supported by NIH Research Career Award K23 DK65978-04. The investigators gratefully acknowledge the commitment and dedication of all participants in the DPP, without whom this work would not have been possible.

Duality of interest The authors declare that there is no duality of interest associated with this manuscript.

\section{References}

1. Barroso I (2005) Genetics of type 2 diabetes. Diabet Med 22:517-535

2. Inoue H, Tanizawa Y, Wasson J et al (1998) A gene encoding a transmembrane protein is mutated in patients with diabetes mellitus and optic atrophy (Wolfram syndrome). Nat Genet 20:143-148

3. Sandhu MS, Weedon MN, Fawcett KA et al (2007) Common variants in WFS1 confer risk of type 2 diabetes. Nat Genet 39: 951-953

4. The Diabetes Prevention Program Research Group (2002) Reduction in the incidence of type 2 diabetes with lifestyle intervention or metformin. N Engl J Med 346:393-403

5. Florez JC, Jablonski KA, Kahn SE et al (2007) Type 2 diabetesassociated missense polymorphisms KCNJ11 E23K and ABCC8 A1369S influence progression to diabetes and response to interventions in the Diabetes Prevention Program. Diabetes 56:531-536

6. Hsieh FY, Lavori PW (2000) Sample-size calculations for the Cox proportional hazards model with nonbinary covariates. Control Clin Trials 21:552-560

7. Diabetes Genetics Initiative of Broad Institute of Harvard and MIT, Lund University and Novartis Institutes for BioMedical Research (2007) Genome-wide association analysis identifies loci for type 2 diabetes and triglyceride levels. Science 316:1331-1336

8. Zeggini E, Weedon MN, Lindgren CM et al (2007) Replication of genome-wide association signals in U.K. samples reveals risk loci for type 2 diabetes. Science 316:1336-1341

9. Frayling TM (2007) Genome-wide association studies provide new insights into type 2 diabetes aetiology. Nat Rev Genet 8:657-662

10. Franks PW, Rolandsson O, Debenham SL et al (2007) Replication of the association between variants in WFS1 and risk of type 2 diabetes in European populations. Diabetologia DOI 10.1007/ s00125-007-0887-6 